\title{
Thermodynamics and entropy of self-gravitating matter shells and black holes in $d$ dimensions
}

\author{
Rui André, José P. S. Lemos, ${ }^{\dagger}$ and Gonçalo M. Quinta \\ Centro de Astrofísica e Gravitação - CENTRA, Departamento de Física, Instituto Superior Técnico - IST, \\ Universidade de Lisboa - UL, Av. Rovisco Pais 1, 1049-001 Lisboa, Portugal
}

(Received 23 February 2019; published 25 June 2019)

\begin{abstract}
The thermodynamic properties of self-gravitating spherical thin matter shells and black holes in $d>4$ dimensions are studied, extending previous analysis for $d=4$. The shell joins a Minkowski interior to a Tangherlini exterior, i.e., a Schwarzschild exterior in $d$ dimensions with $d \geq 4$. The junction conditions and the first law of thermodynamics enable one to establish that the entropy of the thin shell depends only on its own gravitational radius. Endowing the shell with a power-law temperature equation of state allows one to determine a precise form for the entropy and to perform a thermodynamic stability analysis for the shell. An interesting case is when the shell's temperature has the Hawking form, i.e., it is inversely proportional to the shell's gravitational radius. It is shown in this case that the shell's heat capacity is positive, and thus there is stability, for shells with radii in between their own gravitational radius and the radius of circular photonic orbits, unexpectedly reproducing York's thermodynamic stability criterion for a $d=4$ black hole in the canonical ensemble. Moreover, the Euler relation for the matter shell is derived, the Bekenstein and holographic entropy bounds are studied, and the large $d$ limit is analyzed. Within this formalism the thermodynamic properties of black holes can be studied, too. Putting the shell at its own gravitational radius, i.e., at the black hole stage, obliges one to choose precisely the Hawking temperature for the shell which in turn yields the Bekenstein-Hawking entropy. The stability analysis implies that the black hole is thermodynamically stable, substantiating that in this configuration our system and York's canonical ensemble black hole are indeed the same system. In addition, within this formalism the Smarr formula for black holes in $d$ dimensions appears naturally and surprisingly.
\end{abstract}

DOI: 10.1103/PhysRevD.99.125013

\section{INTRODUCTION}

Black holes are thermodynamics systems that have an internal energy [1,2], an entropy [3], and a temperature [4]. A statistical physics thermodynamic treatment can be given through a path integral approach [5], and in a consistent manner black holes can be put in a canonical ensemble by defining a temperature for a heat bath in a given region of space [6-8]. These works were performed for Schwarzschild and Reissner-Nordström black holes in four dimensions.

Self-gravitating matter systems also possess thermodynamic properties. Perhaps, the simplest self-gravitating matter system is a thin shell. Thermodynamic studies of

\footnotetext{
*rui.andre@tecnico.ulisboa.pt

joselemos@tecnico.ulisboa.pt

"goncalo.quinta@tecnico.ulisboa.pt
}

Published by the American Physical Society under the terms of the Creative Commons Attribution 4.0 International license. Further distribution of this work must maintain attribution to the author(s) and the published article's title, journal citation, and DOI. Funded by SCOAP ${ }^{3}$. thin shells in Schwarzschild and Reissner-Nordström four-dimensional spacetimes have been performed in Refs. [9-12], in which the entropy and the stability of the shells were displayed.

Since black holes and self-gravitating matter systems are thermodynamic systems, it is natural to mix both. This has been done by putting the combined system of black hole plus matter in a canonical ensemble [13]. One can also conceive of a black hole surrounded by a thin shell and study the compound system thermodynamically $[14,15]$. One can then collapse the matter into the initial black hole. The collapse should be done quasistatically and in thermodynamic equilibrium so that the whole setup makes sense thermodynamically. Yet another way is to suppose no initial black hole and some initial self-gravitating matter in thermal equilibrium, for instance, the thin shells considered in Refs. [9-12]. Suppose then the shell gravitationally collapses again quasistatically up to its own gravitational radius, i.e., up to the formation of a black hole. On the verge of the black hole formation, the matter entropy must change in order to give rise to the final black hole entropy [10-12]. In this way, one can test how matter entropy transforms into 
black hole entropy; see also Refs. [16]. For a selfgravitating matter continuum, a generic spacetime matter structure that includes thin shells, one can also address the entropy when the matter is forming a black hole, a situation that has been fully developed within the quasiblack hole formalism [17,18]. An analogous procedure to find black hole properties is the membrane paradigm approach [19-21].

It is surely interesting to see if the thermodynamic properties for black holes and self-gravitating matter are reproduced in dimensions different from 4 and in spacetimes with a cosmological constant. In three dimensions, thermodynamic properties of thin shells in BTZ nonrotating and rotating spacetimes have been studied [22-25] with results that, even in one lower dimension and with the inclusion of a cosmological constant and rotation, somehow repeat the four-dimensional results, confirming that the BTZ spacetime is a good test bed for four-dimensional general relativity. On the other hand, the study of higher $d$-dimensional self-gravitating shells has not been performed. Since there is the intriguing possibility that the Universe has higher hidden dimensions that might be large or small, it is interesting to study how shells and black holes and their thermodynamics properties develop in higher $d>4$ dimensions. Here, we make a thermodynamic study of shells for which the inner spacetime is spherically symmetric Minkowski and the outer spacetime is a Tangherlini spacetime, i.e., a Schwarzschild spacetime in $d$ dimensions, $d \geq 4$. We also take the self-gravitating $d$-dimensional shell to its own gravitational radius and obtain the thermodynamic properties of a $d$-dimensional black hole, such as its entropy, its stability, and the corresponding Smarr formula.

We use known results in $d$ dimensions. For particle orbits in $d$ dimensions, see Ref. [26]; for the Hawking temperature in $d$ dimensions, see Ref. [27]; and for the Smarr formula in $d$ dimensions, see Ref. [28]. We adopt the thermodynamic formalism presented in Ref. [29]. We also study the Bekenstein [30] and the holographic [31-33] entropy bounds for the $d$-dimensional shells. We benefit from the result given in Ref. [34], in which it is shown that to be divergent free quantically black holes must be at the Hawking temperature.

The paper is organized as follows. In Sec. II, the $d$ dimensional interior Minkowski and exterior Schwarzschild, or Tangherlini, metrics are given, and the mechanical properties of the self-gravitating thin shell that makes the junction of the two spacetimes are found. The thermodynamic properties of the shell are prescribed, the first law of thermodynamics applied to the shell is studied, and a generic expression for the entropy of the shell is found. In Sec. III, it is assumed a power-law equation of state for the temperature, an analysis of the local thermodynamic stability is performed, the Euler relation between the relevant thermodynamic quantities is found, the holographic and Bekenstein entropy bounds are studied, and the large $d$ limit is considered. In Sec. IV, the black hole limit is taken, and its properties follow. In Sec. V, conclusions are drawn.

\section{MECHANICS AND THERMODYNAMICS OF SELF-GRAVITATING STATIC THIN SHELLS IN $d$ DIMENSIONS}

\section{A. Mechanics of static thin shells: ADM and rest masses and the equation of state for the pressure}

We write the Einstein field equation in $d$ dimensions in the form

$$
G_{a b}=8 \pi T_{a b},
$$

where $a, b$ are spacetime indices that run from 0 to $d-1$, $G_{a b}$ is the Einstein tensor, $T_{a b}$ the energy-momentum tensor, and it is clear that with this choice for Eq. (1) the $d$-dimensional Einstein field equation has the same form as the four-dimensional one. We put the $d$-dimensional gravitational constant $G_{d}$ to 1 and the speed of light $c$ to 1 .

Consider a spherically symmetric timelike $(d-1)$ hypersurface $\Sigma$ that partitions a $d$-dimensional spacetime into two regions. The region on the inside is denoted by an $\mathrm{i}$ subscript sign, and the outside region is denoted with an o subscript. The partition is given by a thin shell, and we assume that the inside is a $d$-dimensional flat metric with $d \geq 4$ and the outside is a Tangherlini, or $d$-dimensional Schwarzschild with $d \geq 4$, metric.

On the inside, the coordinates are $x_{\mathrm{i}}^{\alpha}=\left(t_{\mathrm{i}}, r, \theta_{1}, \ldots, \theta_{d-2}\right)$, where $t_{\mathrm{i}}$ is the time coordinate inside, $r$ is the radial coordinate, and $\theta_{k}$ are the angular coordinates on a $(d-2)$ dimensional sphere. The metric on the flat inside is thus

$$
d s_{\mathrm{i}}^{2}=-F_{\mathrm{i}} d t_{\mathrm{i}}^{2}+\frac{d r^{2}}{F_{\mathrm{i}}}+r^{2} d \Omega^{2},
$$

with

$$
F_{\mathrm{i}}=1 \text {, }
$$

and

$$
d \Omega^{2}=d \theta_{1}^{2}+\sum_{k=2}^{d-2}\left(\prod_{j=1}^{k-1} \sin ^{2} \theta_{j}\right) d \theta_{k}^{2}
$$

is the line element on a $(d-2)$-dimensional sphere.

On the outside, the coordinates are $x_{\mathrm{o}}^{\alpha}=\left(t_{0}, r, \theta_{1}\right.$, $\left.\ldots, \theta_{d-2}\right)$, where $t_{\mathrm{o}}$ is the time coordinate outside, $r$ is the radial coordinate, and $\theta_{k}$ are the angular coordinates. The metric on the Tangherlini outside is thus

$$
d s_{\mathrm{o}}^{2}=-F_{\mathrm{o}} d t_{\mathrm{o}}^{2}+\frac{d r^{2}}{F_{\mathrm{o}}}+r^{2} d \Omega^{2},
$$


with

$$
F_{\mathrm{o}}=1-\frac{\gamma m}{r^{d-3}},
$$

where

$$
\gamma \equiv \frac{16 \pi}{(d-2) \Omega_{d-2}},
$$

and

$$
\Omega_{d-2}=\frac{2 \pi^{(d-1) / 2}}{\Gamma\left(\frac{d-1}{2}\right)},
$$

$d \Omega^{2}$ is the same line element on a $(d-2)$-dimensional sphere as in Eq. (4), $m$ is the spacetime ADM mass, and $\Gamma$ is the gamma function. In $d=4$, one has $\Gamma(3 / 2)=\sqrt{\pi} / 2$, $\Omega_{2}=4 \pi$, and $\gamma=2$. The spacetime gravitational radius is

$$
r_{+}=(\gamma m)^{1 /(d-3)} .
$$

In $d=4$, one recovers $r_{+}=2 m$. It is useful to define the gravitational area $A_{+}$given by

$$
A_{+}=\Omega_{d-2} r_{+}^{d-2} .
$$

If the spacetime is a black hole spacetime, then $r_{+}$and $A_{+}$ are the horizon radius and the horizon area of the black hole, respectively. There is an additional radius that pops out naturally in our context. This is the radius of the photon sphere [26]

$$
r_{\mathrm{ph}}=\left(\frac{d-1}{2}\right)^{\frac{1}{d-3}} .
$$

For $d=4$, it gives $3 m$, and recall that $r_{\mathrm{ph}}=3 \mathrm{~m}$ is the photon sphere, where photons can have circular trajectories in the Schwarzschild spacetime. The generalization of the photon sphere radius to $d$ dimensions is indeed Eq. (11) [26].

The self-gravitating shell is at the hypersurface $\Sigma$ defined by

$$
r=R
$$

Letting $\tau$ be the proper time on the shell, the shell's evolution is parametrized as $R(\tau),\left.T_{\mathrm{i}}(\tau) \equiv t_{\mathrm{i}}\right|_{\Sigma}$, and $\left.T_{\mathrm{o}}(\tau) \equiv t_{\mathrm{o}}\right|_{\Sigma}$. Define the metric and coordinates on the shell by $h_{a b}$ and $y^{a}=\left(\tau, \theta_{1}, \ldots, \theta_{d-2}\right)$, respectively, such that on the shell the line element $d s_{\Sigma}^{2}=h_{a b} d y^{a} d y^{b}$ is

$$
d s_{\Sigma}^{2}=-d \tau^{2}+R^{2}(\tau) d \Omega^{2} .
$$

The first junction condition demands continuity of the metric across the shell. This is obtained by assuring $\left[h_{a b}\right]=0$, where square brackets [] denote the jump in the quantity across the hypersurface. The first junction condition then yields $-\dot{T}_{\mathrm{i}}^{2}+\dot{R}^{2}=-F_{\mathrm{o}} \dot{T}_{\mathrm{o}}{ }^{2}+\frac{\dot{R}^{2}}{F_{\mathrm{o}}}=-1$, where a dot denotes differentiation with respect to $\tau$ and we have used $F_{\mathrm{i}}=1$. We can now proceed to the second junction condition. The shell is assumed to be a perfect fluid, so its stress tensor is given by $S_{a b}=(\sigma+p) u_{a} u_{b}+$ $p h_{a b}$, where $\sigma$ is the rest energy density, $p$ is the tangential pressure acting on the $(d-2)$-sphere at radius $R$, and $u^{a}$ is the fluid's $d$-velocity. Denoting the rest mass of the shell by $M$, one has that the relation between $M$, the area $A$ of the shell, and $\sigma$ is

$$
M=\sigma A,
$$

where $A$ is

$$
A=\Omega_{d-2} R^{d-2} .
$$

The second junction condition is $S_{a b}=-\frac{1}{8 \pi}\left(\left[K_{a b}\right]-[K] h_{a b}\right)$, with $K_{a b}$ and $K$ standing for the extrinsic curvature and its trace, respectively. The static case is characterized by $\ddot{R}=\dot{R}=0$. The junction then yields

$$
\begin{gathered}
m=M-\frac{\gamma M^{2}}{4 R^{d-3}}, \\
p=\frac{(d-3) \gamma M^{2}}{4(d-2) \Omega_{d-2} R^{d-2}\left(R^{d-3}-\frac{\gamma M}{2}\right)} .
\end{gathered}
$$

The shell can surely be put at infinity, $R=\infty$. On the other hand, the static shell concept only makes sense if the radius of the shell $R$ bounds from above the spacetime gravitational radius $r_{+}$. For $R=r_{+}$, the shell turns into a black hole. For $R<r_{+}$, there is no static shell. Thus, $R$ obeys

$$
R \geq r_{+},
$$

with the inequality being valid up to infinity. The redshift function $k$ at the shell's position $R$ is a quantity that appears quite often. It is defined by

$$
k=\sqrt{1-\left(\frac{r_{+}}{R}\right)^{d-3}} .
$$

We see from Eqs. (18) and (19) that

$$
0 \leq k \leq 1
$$

We can then put the rest mass $M$ and the tangential pressure $p$ given in Eqs. (16) and (17), respectively, in terms of the redshift function $k$ given in Eq. (19) to find

$$
M=\frac{2 R^{d-3}}{\gamma}(1-k),
$$




$$
p=\frac{(d-3)(1-k)^{2}}{16 \pi R k}
$$

\section{B. Thermodynamics on the shell: First law, functional form of the temperature equation of state, and entropy}

Consider the self-gravitating thin shell to be thermally isolated; i.e., it is an adiabatic system. In any infinitesimal neighborhood of a point in the shell, one defines a local temperature $T$ at the shell, a local entropy density $s$, a local rest mass density $\sigma$, a local tangential pressure $p$, and a local element area $a$. The first law of thermodynamics for this small region in the shell is $T d s=d \sigma+p d a$. This can be integrated on angles at radius $R$ to give the first law of thermodynamics for the shell

$$
T d S=d M+p d A
$$

where $S$ is its entropy, $M$ is its rest mass, $p$ is the tangential pressure, and $A$ is its area. We work in the entropy representation [29], i.e., we consider $S$ as function of $M$ and $A$,

$$
S=S(M, A)
$$

The temperature $T$ and the pressure $p$ are given by equations of state $T=T(M, A)$ and $p=p(M, A)$, respectively. The equation of state for the temperature $T=$ $T(M, A)$ is free and has to be specified. The equation of state for the pressure $p=p(M, A)$ is imposed on us through the junction conditions and is given by Eq. (17) or Eq. (22) with the help of Eq. (15). Both $T(M, A)$ and $p(M, A)$ are formally given by $T(M, A)=\left(\frac{\partial S}{\partial M}\right)_{A}$ and $p(M, A)=\left(\frac{\partial S}{\partial A}\right)_{M}$. It is useful to define the inverse temperature $\beta$,

$$
\beta=\frac{1}{T},
$$

where also $\beta=\beta(M, A)$. Equation (23) is then $d S=\beta d M+\beta p d A$, and it can only be an exact differential for the entropy if the integrability condition

$$
\left(\frac{\partial \beta}{\partial A}\right)_{M}=\left(\frac{\partial(\beta p)}{\partial M}\right)_{A}
$$

is satisfied. Then, given $\beta=\beta(M, A)$ and $p=p(M, A)$ obeying Eq. (26), $S=S(M, A)$ in Eq. (24) can be determined explicitly by integration.

Using Eqs. (9), (15), and (16), we can make the thermodynamic variable change $(M, A) \rightarrow\left(r_{+}, R\right)$ and upon using Eq. (17) or Eq. (22) find that the differential for the entropy is given solely by a differential on the gravitational radius $r_{+}$, with Eq. (23) taking the form

$$
d S=\beta\left(r_{+}, R\right) \frac{d-3}{\gamma k} r_{+}^{d-4} d r_{+} .
$$

In terms of $\left(r_{+}, R\right)$, the integrability condition (26) reads

$$
\left(\frac{\partial \beta}{\partial R}\right)_{r_{+}}=\beta \frac{(d-3)\left(1-k^{2}\right)}{2 k^{2} R},
$$

which has for solution an inverse temperature Tolman formula at the shell's location, i.e.,

$$
\beta\left(r_{+}, R\right)=b\left(r_{+}\right) k\left(r_{+}, R\right),
$$

where $b\left(r_{+}\right)$is an arbitrary function of $r_{+}$alone. Since $k \rightarrow 1$ as $R \rightarrow \infty, b$ provides the inverse temperature if the shell were placed at infinity. An alternative interpretation is to consider the Tolman redshift formula. Suppose that there is some negligible but effective leaking in the form of radiation from the thermally isolated shell to infinity. From the Tolman formula, we have that the inverse temperature at a given radius $r$ of the leaked radiation is given by $\beta\left(r_{+}, r\right)=b\left(r_{+}\right) k\left(r_{+}, r\right)$. At infinity, $k\left(r_{+}, \infty\right)=1$, and so the inverse temperature of the radiation there is $b\left(r_{+}\right)$. Now, inserting Eq. (29) into the entropy differential equation (27), one gets $d S=\frac{d-3}{\gamma} b\left(r_{+}\right) r_{+}^{d-4} d r_{+}$. Thus, the total entropy of the shell is given by the sum of all the entropy differentials up to that $r_{+}$, i.e.,

$$
S\left(r_{+}\right)=\frac{d-3}{\gamma} \int_{0}^{r_{+}} b(r) r^{d-4} d r .
$$

In Eq. (30), the integration constant is fixed under the condition that $S(0)=S_{0}$, and we put $S_{0}=0$. Equation (30) provides the equation for the shell's entropy for any acceptable equation of state for $b\left(r_{+}\right)$, and it shows that the entropy does not depend on the shell radius $R$; it depends only on the gravitational radius $r_{+}$. In other words, shells with different radius $R$ but with the same $r_{+}$have the same entropy. This is a known, but nevertheless striking, result.

\section{SHELLS WITH A POWER-LAW EQUATION OF STATE IN $\boldsymbol{d}$ DIMENSIONS: ENTROPY, LOCAL THERMODYNAMIC STABILITY, EULER RELATION, ENTROPY BOUNDS, AND LARGE $d$}

\section{A. Entropy of a shell with a temperature power-law equation of state}

We still have the freedom to choose the equation of state for the inverse temperature of the shell given in the function $b\left(r_{+}\right)$. To proceed, we assume as equation of state for $b\left(r_{+}\right)$a power-law function of the form

$$
b\left(r_{+}\right)=4 \pi \eta \frac{a+1}{d-3} r_{+}^{a(d-2)+1},
$$


where $\eta$ and $a$ are free parameters without units and $\frac{a+1}{d-3}$ appears for convenience. We have put the Boltzmann constant equal to 1 so that temperature has units of mass. We also put the Planck constant $\hbar$ equal to 1 . Then, the Planck length for a $d$-dimensional spacetime $l_{p}=\left(\frac{G_{d} \hbar}{c^{3}}\right)^{\frac{1}{d-2}}$ is 1 , and the Planck mass $m_{p}=\left(\frac{c^{5-d} \hbar^{d-3}}{G_{d}}\right)^{\frac{1}{d-2}}$ is also 1; i.e., all quantities are measured in Planck units. The choice in Eq. (31) for $b\left(r_{+}\right)$is analogous to the choice in Ref. [9]. Note that the case $a=0$ is of particular interest as the inverse temperature has the inverse Hawking temperature form; it is proportional to $r_{+}$[see Eq. (31)]. If further we choose $\eta=1$, then $b\left(r_{+}\right)=\frac{4 \pi}{d-3} r_{+}$, and the shell has precisely the Hawking temperature in $d$ dimensions [27].

Putting Eq. (31) into Eq. (30) leads to the following expression for the entropy of the self-gravitating shell:

$$
S=\frac{1}{4} \eta \Omega_{d-2} r_{+}^{(a+1)(d-2)} .
$$

From this expression, note that

$$
a>-1
$$

otherwise, the entropy would diverge in the limit $r_{+} \rightarrow 0$, a situation we avoid. The case $a=0$ that has the Hawking inverse temperature form yields an entropy proportional to it is proportional to $r_{+}^{d-2}$, i.e., proportional to $A_{+}$and so has the Bekenstein-Hawking form.

\section{B. Intrinsic thermodynamic stability}

\section{Generics}

Following Ref. [29], one can analyze thermodynamic local stability of a system in relation to the entropy fundamental equation $S=S(M, A)$. Stable solutions are considered using Le Chatelier's principle, which states that a stable system will tend to restore its equilibrium homogeneity state when a small nonhomogeneous change is performed on it. The thin matter shell solution is a good approximation to a layer of matter with a very small thickness. Let us divide this layer into an inner layer and an outer layer with proper mass $M$, say, each and with no thermic contact. The fundamental equation for each layer is $S=S(M, A)$. So, the initial entropy of the total system is $2 S(M, A)$. Now, remove some mass $\Delta M$ from one subsystem to the other, and get for the entropy of total system $S(M+\Delta M, A)+S(M-\Delta M, A)$. If the thermic contact is removed, heat flows from one side to the other. By the second law of thermodynamics the entropy should increase and for the system to be stable it should return to its original value $2 S(M, A)$. So, $2 S(M, A) \geq S(M+\Delta M, A)+S(M-\Delta M, A)$. Taking the limit $\Delta M \rightarrow 0$ stability means

$$
\left(\frac{\partial^{2} S}{\partial M^{2}}\right)_{A} \leq 0
$$

The heat capacity is given by $C_{A} \equiv\left(\frac{\partial M}{\partial T}\right)_{A}=-T^{-2}\left(\frac{\partial^{2} S}{\partial M^{2}}\right)_{A}^{-1}$. So, Eq. (34) is equivalent to requiring a positive heat capacity at constant area $C_{A}$.

Analogously, one can consider the thermodynamic stability in relation to the area $A$ and obtain

$$
\left(\frac{\partial^{2} S}{\partial A^{2}}\right)_{M} \leq 0
$$

For a small change of both $M$ and $A$ simultaneously, the stability condition is

$$
\left(\frac{\partial^{2} S}{\partial M^{2}}\right)\left(\frac{\partial^{2} S}{\partial A^{2}}\right)-\left(\frac{\partial^{2} S}{\partial M \partial A}\right)^{2} \geq 0 .
$$

Note that one can analyze each condition at a time. Condition (34) is the actual stability condition if the selfgravitating shell is held at fixed $A$, i.e., at fixed radius $R$. Condition (35) is the actual stability condition if the shell is held at fixed proper mass $M$. If the area $A$ and the proper mass $M$ are not fixed, then condition (36) also counts, and one needs to check it.

\section{Stability for free proper mass $M$ and at fixed area $A$, i.e., at fixed shell radius $R$}

Condition (34) is the stability condition if the proper mass $M$ is free to change and the shell is held at fixed area $A$, i.e., fixed radius $R$. Since the heat capacity is given by $C_{A} \equiv\left(\frac{\partial M}{\partial T}\right)_{A}=-T^{-2}\left(\frac{\partial^{2} S}{\partial M^{2}}\right)_{A}^{-1}$, Eq. (34) is equivalent to requiring a positive heat capacity at constant area $C_{A}$, i.e.,

$$
C_{A} \geq 0 .
$$

Equation (32) together with Eqs. (9) and (16) yields $\left(\frac{\partial^{2} S}{\partial M^{2}}\right)_{A}=\frac{2(1+a)(d-2) S(M, A)}{(d-3)^{2} M^{2}(1-k)^{2}}\left[k^{2}(d-1+2 a(d-2))-(d-3)\right]$. Thus, Eq. (34) or Eq. (37) gives

$$
k^{2}(d-1+2 a(d-2))-(d-3) \leq 0 .
$$

If $a \leq-(d-1) /(2(d-2))$, Eq. (38) is always satisfied. Since we have imposed $a>-1$, Eq. (33), we have for $-1<a \leq-(d-1) /(2(d-2))$ that Eq. (38) is satisfied. On the other hand, for $a>-(d-1) /(2(d-2))$, Eq. (38) is satisfied when $-k_{1} \leq k \leq k_{1}$, with $k_{1}=\sqrt{\frac{d-3}{d-1+2 a(d-2)}}$. Since we have $0 \leq k$ [recall Eq. (19)], this condition can be rewritten as $0 \leq k \leq k_{1}$. Now, note that the expression inside the square root in $k_{1}$, i.e., $\frac{d-3}{d-1+2 a(d-2)}$, is always greater than 1 if $a<-1 /(d-2)$. Then, since $k \leq 1$ [recall again Eq. (19)], we have that from the equation above, if $a \leq-1 /(d-2)$, Eq. (38) is always satisfied. Since we found that for $-1<a \leq-(d-1) /(2(d-2)) \mathrm{Eq}$. (38) is 
satisfied, we can extend this range to $-1<a \leq$ $-1 /(d-2)$. Now, for $a>-1 /(d-2)$, the expression inside the square root in $k_{1}$, i.e., $\frac{d-3}{d-1+2 a(d-2)}$, is always smaller than 1 . This imposes a requirement on $k$ indeed, i.e., $k \leq k_{1}$, with $k_{1} \leq 1$.

In brief, stability for free proper mass $M$ and at constant area $A$ means that Eq. (34) holds, which in turn means that Eq. (37) also holds; i.e., the heat capacity $C_{A}$ is positive, $C_{A} \geq 0$. Specifically, we found that for free $M$ and fixed $A$,

$$
\text { stability always for }-1<a \leq-\frac{1}{d-2} \text {, }
$$

and Eq. (38) is satisfied when $0 \leq k \leq k_{1}$ for $-1 /(d-2)<a<\infty$, i.e.,

stability when $0 \leq k \leq k_{1}, \quad$ for $-\frac{1}{d-2}<a<\infty$,

with

$$
k_{1}=\sqrt{\frac{d-3}{d-1+2 a(d-2)}} .
$$

Note anew that $0<k_{1} \leq 1$ in this case, i.e., for $-1 /(d-2)<a$. Using Eq. (19), we can put condition (40) in terms of $R / r_{+}$,

$$
1 \leq \frac{R}{r_{+}} \leq \frac{1}{\left(1-k_{1}^{2}\right)^{\frac{1}{d-3}}}
$$

for $-1 /(d-2)<a<\infty$. Changing from $r_{+}$to $M$ through Eqs. (9) and (16), we obtain that the thin shell's radius is bounded from above as $\frac{2 R^{d-3}}{\gamma M} \leq \frac{1}{1-k_{1}}$.

The case $a=0$ is of particular interest as the inverse temperature has the inverse Hawking temperature form; it is proportional to $r_{+}$[see Eq. (31)]. Putting $a=0$ in $k_{1}$ [see Eq. (41)], we get $k_{1}=\sqrt{\frac{d-3}{d-1}}$, and the stability is given then by Eq. (40). One can solve for $\frac{R}{r_{+}}$[see also Eq. (42)], to obtain $1 \leq \frac{R}{r_{+}} \leq\left(\frac{d-1}{2}\right)^{\frac{1}{d-3}}$. Looking at Eq. (11), we see this is

$$
r_{+} \leq R \leq r_{\mathrm{ph}}
$$

i.e., the shell is thermodynamically stable if its radius $R$ is in between the gravitational radius $r_{+}$and the photon sphere radius $r_{\mathrm{ph}}$. For $d=4$, Eq. (43) is $r_{+} \leq R \leq \frac{3}{2} r_{+}$, and putting $r_{+}=2 m$, one gets $2 m \leq R \leq 3 m$. This is a striking outcome as it reminds one of York's result for the thermal stability of the $d=4$ black hole in the canonical ensemble [6]. York's approach implies that for a Scharzschild black hole in a heat reservoir of fixed radius $R$ at temperature $T$, i.e., in a canonical ensemble, the heat capacity of the black hole system is positive only if $2 m \leq R \leq 3 m$, so in this
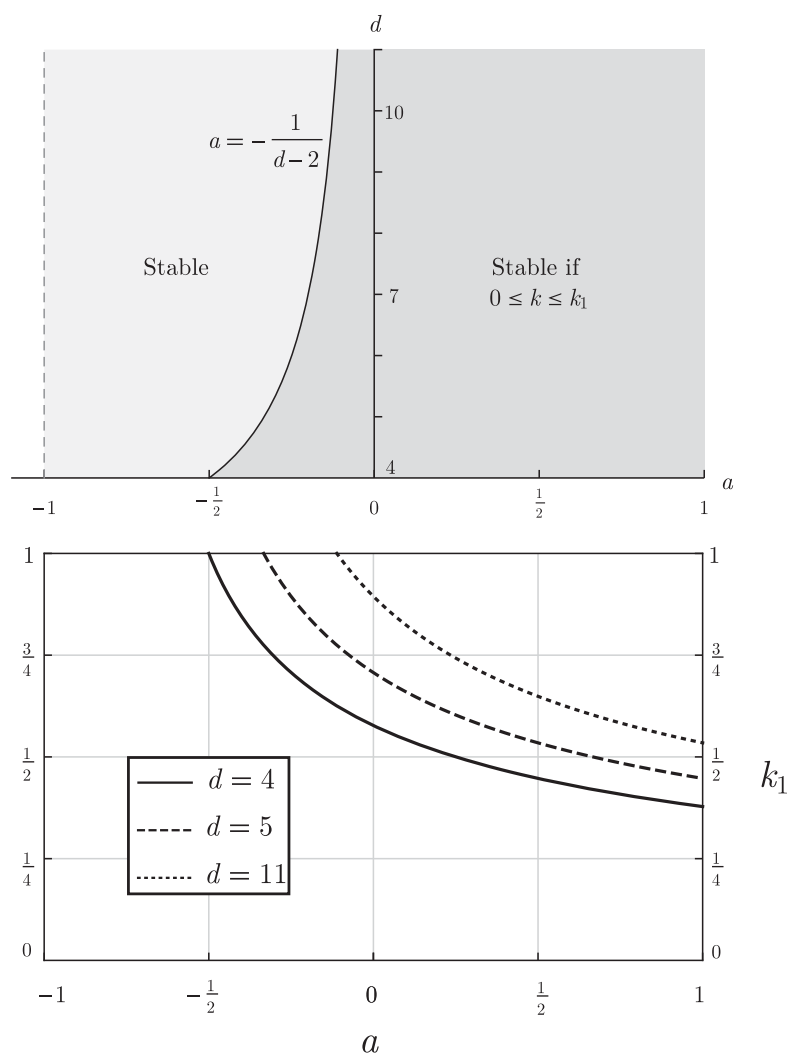

FIG. 1. Intrinsic stability of the shell for free proper mass $M$ and at fixed area $A$, i.e., fixed radius $R$, given in Eqs. (39) and (40), for a self-gravitating shell with a power-law temperature equation of state. Stability at fixed area $A$ is equivalent to having a heat capacity $C_{A}$ obeying $C_{A} \geq 0$. Top: The stability regions in the parameter space given by the equation of state exponent $a$ vs the number of dimensions $d$, an integer number with $d \geq 4$, are displayed. In the region $a \leq-\frac{1}{d-2}$, the shell is always stable. In the region $a>-\frac{1}{d-2}$, the shell is only stable if $0 \leq k \leq k_{1}$. Bottom: Plot of $k_{1}$ given in Eq. (41) as a function of $a$ for three different values of $d, d=4,5,11$. The shell is stable if $k$ is below the respective $k_{1}$. See the text for details.

range, the system is stable. Our result says that for $a=0$, the heat capacity is positive if the shell is in the range $2 m \leq R \leq 3 m$. The shell's heat capacity is measured for $A$ fixed, i.e., $R$ fixed, and the shell itself acts as a heat reservoir. The two systems thus have similarities but are different. One is a black hole in a heat reservoir at temperature $T$ and the Schwarzschild solution holds inside that reservoir, the other is a massive shell at temperature $T$ and the Minkowski solution holds inside that shell. This is an unexpected result and hints that what is important for thermodynamic stability is the place of the shell alone, whether it is a heat reservoir massless shell or a massive shell.

In Fig. 1, we plot the stability regions in the parameter space given by the equation of state exponent $a$ vs the number of dimensions $d$, an integer number with $d \geq 4$. Adjacently, we also plot the quantity $k_{1}$ given in Eq. (41) in 
terms of the equation of state exponent $a$ for three different dimensions $d, d=4,5,11$. This makes it easier to follow the stability parameters.

\section{Stability for fixed proper mass $M$ and for free area $A$}

Condition (35) is the stability condition if the proper mass $M$ of the shell is held fixed and the area $A$ is free to change. Equation (32) together with Eqs. (9), (15), and (16) yields $\left(\frac{\partial^{2} S}{\partial A^{2}}\right)_{M}=\frac{(1+a) S(M, A)}{A^{2}(d-2)(1-k)}[(1-k)(2+a)(d-2)-2(2 d-5)]$. Thus, Eq. (35) gives

$-k(d-2)(a+2)-2(2 d-5)+(d-2)(a+2) \leq 0$.

The solution for Eq. (44) is $k \geq k_{2}$ where $k_{2}=\frac{a-2 \frac{d-3}{d-2}}{a+2}$. Recalling from Eq. (33) that $a>-1$, we note that $k_{2} \leq 0$ for $-1<a \leq 2(d-3) /(d-2)$ and, since $0 \leq k \leq 1$, Eq. (44) is always satisfied. For $a>2(d-3) /(d-2)$, we have $0<k_{2}<1$, so Eq. (44) is satisfied if $k_{2} \leq k \leq 1$.

So, in brief, stability for fixed $M$ and free $A$ means that Eq. (35) holds. Specifically, we found that for fixed $M$ and free $A$,

$$
\text { stability always for }-1<a \leq 2 \frac{d-3}{d-2} \text {, }
$$

and Eq. (38) is satisfied when $k_{2} \leq k \leq 1$ for $a>2(d-3) /(d-2)$, i.e.,

$$
\text { stability when } k_{2} \leq k \leq 1 \text { for } a>2 \frac{d-3}{d-2} \text {, }
$$

with

$$
k_{2}=\frac{a-2 \frac{d-3}{d-2}}{a+2} .
$$

Using Eq. (19), we can write condition Eq. (46) in terms of $R / r_{+}$,

$$
\frac{R}{r_{+}} \geq \frac{1}{\left(1-k_{2}^{2}\right)^{\frac{1}{d-3}}}
$$

for $a>2(d-2) /(d-2)$. Changing from $r_{+}$to $M$ through Eqs. (9) and (16), we obtain that the thin shell's radius is bounded from below as $\frac{2 R^{d-3}}{\gamma M} \geq \frac{1}{1-k_{2}}$.

For the particularly interesting case $a=0$, we see from Eq. (45) that the $a=0$ shell is thermodynamically stable for any radius, as the condition is independent of it.

In Fig. 2, we plot the stability regions in the parameter space given by the equation of state exponent $a$ vs the number of dimensions $d$, an integer number with $d \geq 4$. Adjacently, we also plot the quantity $k_{2}$ given in Eq. (47) in terms of the equation of state exponent $a$ for three different
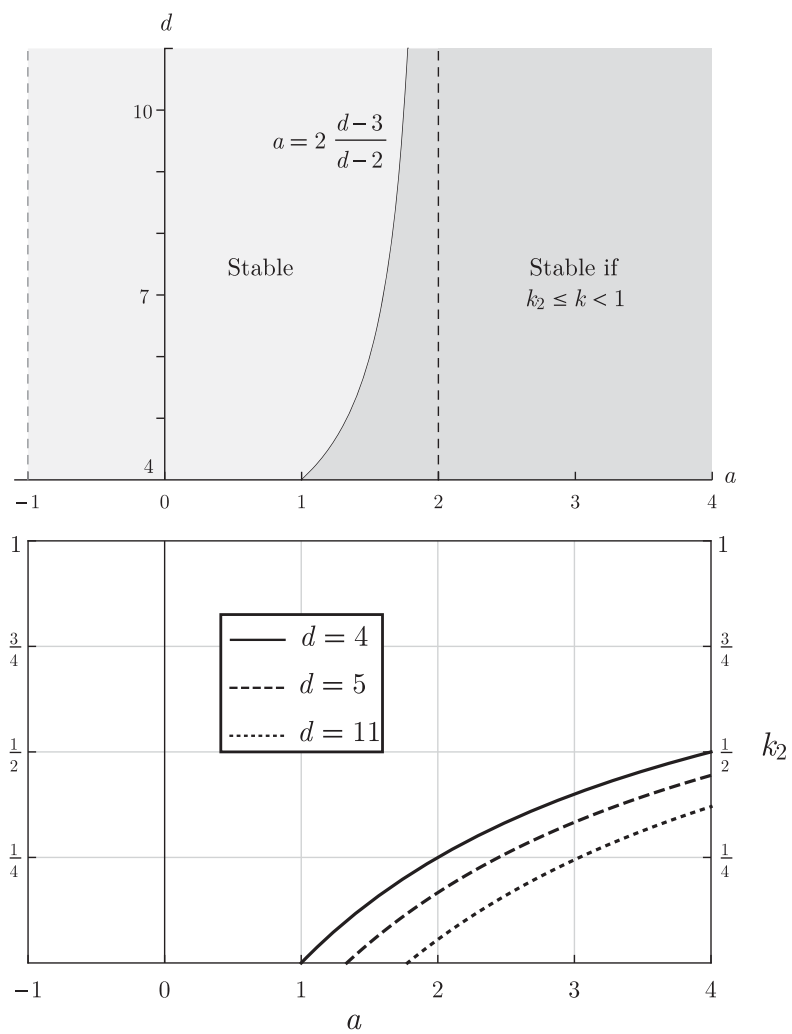

FIG. 2. Intrinsic stability for free area $A$ and fixed proper mass $M$, given in Eqs. (45) and (46), for a self-gravitating shell with a power-law temperature equation of state. Top: The stability regions in the parameter space given by the equation of state exponent $a$ vs the number of dimensions $d$, an integer number with $d \geq 4$, are displayed. In the region $a \leq 2 \frac{d-3}{d-2}$, the shell is always stable. In the region $a>2 \frac{d-3}{d-2}$, the shell is only stable if $k_{2} \leq k \leq 1$. Bottom: Plot of $k_{2}$ as a function of $a$ for three different values of $d, d=4,5,11$. The shell is stable if $k$ lies above the respective $k_{2}$. See the text for details.

dimensions $d, d=4,5,11$. This makes it easier to follow the stability parameters.

\section{Stability for free proper mass $M$ and free area $A$}

In the case $M$ and $A$ are free, condition (36) also counts, and one needs to check it. It will be seen that Eq. (36) yields the most stringent conditions between the three conditions. Equation (32) together with Eqs. (9), (15), and (16) yields $\frac{\partial^{2} S}{\partial M \partial A}=\frac{2(1+a) S(M, A)}{M(d-3)(1-k)}[-(1-k)(1+a)(d-2)+2 d-5+$ $a(d-2)]$. Thus, Eq. (36) gives

$$
\begin{aligned}
& k^{2}(2+a(d-1))+\frac{2 k(d-3)(1+a(d-2))}{d-2} \\
& \quad+a(d-3) \leq 0 .
\end{aligned}
$$

For $-1<a \leq-2 /(d-1)$, the inequality is always satisfied by any $0 \leq k \leq 1$. For $a>-2 /(d-1)$, the inequality is satisfied by $k_{3-} \leq k \leq k_{3}$, where $k_{3-}$ and $k_{3}$ are the roots 
in Eq. (49). Since $k_{3-}<0$, it can be discarded, and the inequality is satisfied by any $0 \leq k \leq k_{3}$, where $k_{3}=-\frac{(d-3)(1+a(d-2))}{(2+a(d-1))(d-2)}+\frac{\sqrt{(d-3)(d-3-2 a(1+a(d-2))(d-2))}}{(2+a(d-1))(d-2)}$. However, for $-2 /(d-1)<a \leq-1 /(d-2)$, note that $k_{3} \geq 1$, so that the inequality is satisfied by any $0 \leq k \leq 1$. For $-1 /(d-2)<a \leq 0$, we have that $0 \leq k_{3}<1$, so that the inequality is satisfied by $0 \leq k \leq k_{3}$. For $a>0$, note that $k_{3}<0$, so the inequality cannot be satisfied.

So, in brief, stability for free $M$ and free $A$ means that Eq. (36) holds. Specifically, we found that for free $M$ and free $A$, the solutions have

$$
\text { stability always for }-1<a \leq-\frac{1}{d-2} \text {, }
$$

and Eq. (49) is satisfied when $0 \leq k \leq k_{3}$ for $-1 /(d-2)<a \leq 0$, i.e.,

stability when $0 \leq k \leq k_{3}$ for $-\frac{1}{d-2}<a \leq 0$

with

$$
\begin{aligned}
k_{3}= & -\frac{(d-3)(1+a(d-2))}{(2+a(d-1))(d-2)} \\
& +\frac{\sqrt{(d-3)(d-3-2 a(1+a(d-2))(d-2))}}{(2+a(d-1))(d-2)} 1,
\end{aligned}
$$

and for $a>0$, there are no thermodynamically stable configurations, i.e.,

$$
\text { no stability for } a>0 \text {. }
$$

Using Eq. (19), Eq. (51) can be written in terms of $R / r_{+}$as

$$
1 \leq \frac{R}{r_{+}} \leq \frac{1}{\left(1-k_{3}^{2}\right)^{\frac{1}{d-3}}}
$$

for $-1 /(d-2)<a \leq 0$. Changing from $r_{+}$to $M$ through Eqs. (9) and (16), we obtain that the thin shell's radius is bounded from above as $\frac{2 R^{d-3}}{\gamma M} \leq \frac{1}{1-k_{3}}$.

For the particularly interesting $a=0$ case, the stability condition is given in Eq. (51). It involves the quantity $k_{3}$ given in Eq. (52), which for $a=0$ gives $k_{3}=0$. This means that to be thermodynamically stable under these perturbations, the radius of the shell $R$ obeys $R=r_{+}$. For $a=0$, this is the only thermodynamic stable case.

In Fig. 3, we plot the stability regions in the parameter space given by the equation of state exponent $a$ vs the number of dimensions $d$, an integer number with $d \geq 4$. Adjacently, we also plot the quantity $k_{3}$ given in Eq. (52) in terms of the equation of state exponent $a$ for three different
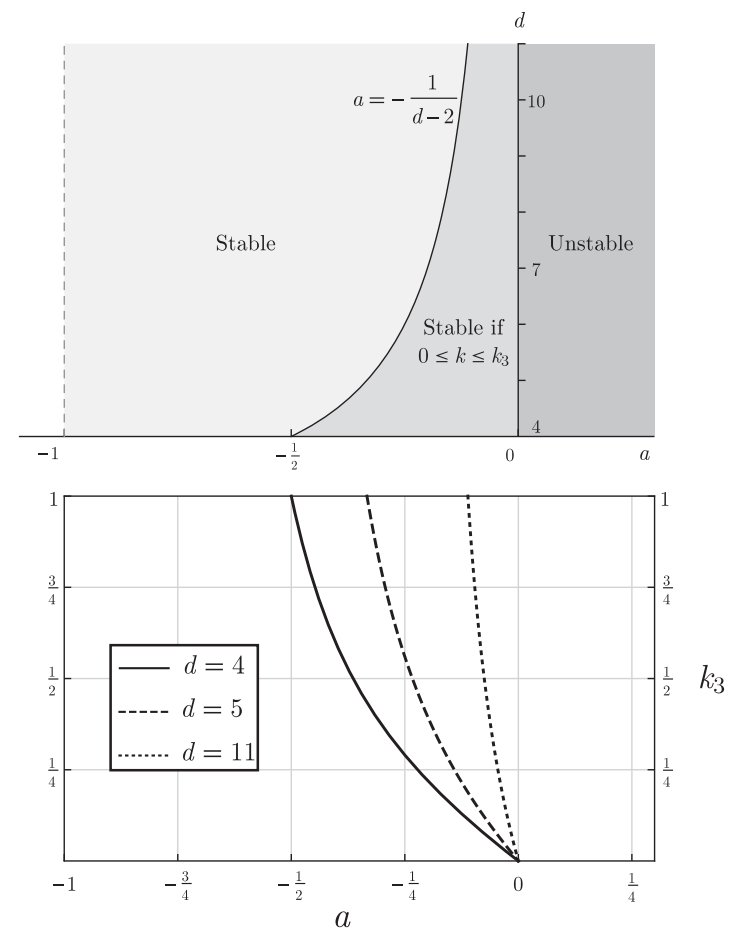

FIG. 3. Intrinsic stability for free proper mass $M$ and free area $A$, given in Eqs. (50), (51), and (53), for a self-gravitating shell with a power-law temperature equation of state. Top: The stability regions in the parameter space given by the equation of state exponent $a$ vs the number of dimensions $d$, an integer number with $d \geq 4$, are displayed. In the region $-1<a \leq-\frac{1}{d-2}$, the shell is always stable. In the region $-\frac{1}{d-2}<a \leq 0$, the shell is only stable if $0 \leq k \leq k_{3}$. In the region $a>0$, the shell never satisfies the thermodynamic stability criterion. Bottom: Plot of $k_{3}$ as a function of $a$ for three different values of $d, d=4,5,11$. The shell is stable if $k$ lies above the respective $k_{3}$. In the region $a>0$ one has $k_{3}<0$ and there is no stability. See the text for details.

dimensions $d, d=4,5,11$. This makes it easier to follow the stability parameters.

\section{Summary of the stability analysis: All three cases together}

Collecting the results for the stability of a self-gravitating shell, we see that the third condition is the stricter one for stability. Indeed: (i) Equations (39) and (50) give the same result and are stricter than (45) in the range of $a$. (ii) In the range $-1 /(d-2)<a \leq 0$ of Eq. (51), $k_{3}<k_{1}$ always, so Eq. (51) makes Eq. (40) spurious in this range of $a$. (iii) In the range $a>0$ of Eq. (53), all solutions are unstable, so Eqs. (40) and (46) are irrelevant in this range. Thus, Eqs. (50)-(53) are the ones necessary and sufficient for intrinsic local thermodynamic stability. Nonetheless, Eqs. (39) and (40) are valid for thermodynamic stability at fixed $A$, and Eqs. (45) and (46) are valid for thermodynamic stability at fixed $M$. 


\section{Euler relation}

From the entropy $S$ in Eq. (32), and using the expression for the ADM mass $m$ in terms of the proper mass $M$ given in Eq. (16), one finds that

$$
\frac{\gamma M}{2 R^{d-3}}=1-\left[\frac{1}{R^{d-3}}\left(\frac{4 S}{\eta \Omega_{d-2}}\right)^{\frac{d-3}{(a+1)(d-2)}}\right]^{\frac{1}{2}} .
$$

Applying Euler's theorem on homogeneous functions [29] to $M$, which is homogeneous of degree $\frac{d-3}{(a+1)(d-2)}$ in $S$ and $\frac{d-3}{d-2}$ in $A$, yields the Euler relation for this system,

$$
M=\frac{(a+1)(d-2)}{d-3} T S-\frac{d-2}{d-3} p A .
$$

From the presence of the free parameter $a$, we see that the Euler relation is dependent on the equation of state for the temperature.

The scaling laws for the self-gravitating shell are $M \rightarrow \lambda M, \quad S \rightarrow \lambda^{\frac{(a+1)(d-2)}{d-3}} S$, and $A \rightarrow \lambda^{\frac{d-2}{d-3}} A$. This makes sense. Indeed, under this rescaling, one has from Eq. (31) $T \rightarrow \lambda^{-1-a(d-2)} T$ and from Eq. (22) $p \rightarrow \lambda^{-\frac{1}{d-3}} p$, which altogether make $M \rightarrow \lambda M$ in Eq. (56).

Taking the differential of Euler's relation, Eq. (56), and taking into account the first law, Eq. (23), one obtains the Gibbs-Duhem relation for this system:

$$
\begin{gathered}
(a+1)(d-2) S d T+(1+a(d-2)) d M \\
+a(d-2) p d A-(d-2) A d p=0 .
\end{gathered}
$$

An interesting case is $a=0$. So, let us put $a=0$ in the equation of state for the shell's temperature. Then, from Eq. (56), we find then that the Euler relation for such a shell reads $M=\frac{d-2}{d-3} T S-\frac{d-2}{d-3} p A$, and the shell's proper mass is a homogeneous function of degree $\frac{d-3}{d-2}$ in $S$ and $A$. The scaling laws for the self-gravitating shell in this $a=0$ case are $M \rightarrow \lambda M, S \rightarrow \lambda^{\frac{d-2}{d-3}} S$, and $A \rightarrow \lambda^{\frac{d-2}{d-3}} A$. Taking the differential of Euler's relation, Eq. (56), and taking into account the first law, Eq. (23), one obtains the Gibbs-Duhem relation for this system $(d-2) S d T+$ $d M-(d-2) A d p=0$.

\section{Other topics on entropy}

\section{Bekenstein entropy bound for the d-dimensional shell}

The Bekenstein bound relates the entropy and the energy of a system. We follow the argument presented by Bekenstein for four dimensions in Ref. [30] and turn it into a $d$-dimensional bound. Given a $d$-dimensional spherical object with energy $E$, size $l$, and entropy $S_{E}$, and a black hole with horizon radius $r_{+}$, area $A_{+}$, and entropy $S_{+}=\frac{1}{4} A_{+}$, one has that the initial entropy $S_{\mathrm{i}}$ of the system is $S_{\mathrm{i}}=\frac{1}{4} A_{+}+S_{E}$. If the object is swallowed by the black hole, this will grow by an area $\Delta A_{+}$, so the final entropy is $S_{\mathrm{f}}=\frac{1}{4}\left(A_{+}+\Delta A_{+}\right)$. From $r_{+}^{d-3}=\gamma m$ [see Eq. (9)] and $A_{+}=\Omega_{d-2} r_{+}^{d-2} \quad$ [see Eq. (10)], we get $\Delta A_{+}=$ $\gamma \Omega_{d-2}\left(\frac{d-2}{d-3}\right) E r_{+}$, where we have naturally put $E=\Delta m$. For the generalized second law of thermodynamics to hold, one must have $S_{\mathrm{i}} \leq S_{\mathrm{f}}$, so then $S_{E} \leq \frac{\gamma \Omega_{d-2}}{4}\left(\frac{d-2}{d-3}\right) E r_{+}$. If $l$ is not small compared to $r_{+}$, then a bound like $S_{E}<$ $\alpha \frac{\gamma \Omega_{d-2}}{4}\left(\frac{d-2}{d-3}\right) E l$ must exist, for some value of $\alpha$ which cannot be set by this argument. We choose $\alpha$ as $\alpha=\frac{d-3}{d-2}$ for reasons given below. Using $\gamma \Omega_{d-2}=\frac{16 \pi}{d-2}$ [see Eq. (7)], the bound is

$$
S_{E} \leq \frac{4 \pi}{d-2} E l .
$$

Now, although the bound was suggested here through a definite example involving matter and a black hole, Eq. (58) is assumed to be valid for all matter in all kinds of situations and is called the Bekenstein bound. In particular, it can be applied to the self-gravitating shells we have been considering.

Let us suppose a self-gravitating shell with energy $E$ and typical length $l$. In the shell case, the quantity $E$ can have two interpretations. It can be interpreted either as the rest mass $M$ of the shell, $E=M$, or as the ADM mass of the spacetime, $E=m$. The length $l$ can be put equal to the radius of the system $l=R$.

For $E=M$, the bound Eq. (58) for the entropy $S$ of the shell is

$$
S \leq \frac{4 \pi}{d-2} M R .
$$

Using Eq. (21) together with Eq. (7), this can be put as $S \leq \frac{1}{2} \Omega_{d-2}(1-k) R^{d-2}$.

For $E=m$, the bound (58) for the entropy $S$ of the shell is

$$
S \leq \frac{4 \pi}{d-2} m R
$$

Using Eq. (9) together with Eq. (19), this yields $S<\frac{1}{4} \Omega_{d-2}\left(1-k^{2}\right) R^{d-2}$.

Which bound shall one choose, the one given in Eq. (59) or the one given in Eq. (60), cannot be settled by this analysis.

\section{Holographic entropy bound for the d-dimensional shell}

The holographic entropy bound [31,32] claims that in a full developed theory of quantum gravity, the entropy $S_{A}$ in a region enclosed by an area $A$ is always less than or equal to $A / 4$ in Planck units,

$$
S_{A} \leq \frac{A}{4}
$$


One insight for the bound came from the gravitational collapse of a star of area $A$ and the entropy law governing black holes [32]. For a black hole, the entropy is precisely equal to one-quarter of its horizon area, so black holes saturate the inequality (61). It is further conjectured that the bound also holds for higher $d$ dimensions, with the area $A$ being a $d-2$ surface $A$ enclosing a $d-1$ volume [33].

In the case in hand, we have a thermodynamic selfgravitating shell with a particular equation of state, Eq. (31). It is thus relevant to know whether the holographic entropy bound is automatically satisfied or if both the junction and stability conditions still allow for configurations the entropy of which exceeds the bound. Since the holographic bound questions the feasibility of a physical system that exceeds it, it is relevant to see how it works for shells.

For the shell's area (15) and the shell's entropy (30), the entropy bound (61) is satisfied if $\eta r_{+}^{(a+1)(d-2)} \leq R^{(d-2)}$, i.e.,

$$
\frac{R}{r_{+}} \geq \eta^{\frac{1}{d-2}} r_{+}^{a} .
$$

Given $a$ and $\eta$, the bound is irrelevant if $\eta^{\frac{1}{d-2}} r_{+}^{a} \leq 1$, since in this case $\frac{R}{r_{+}} \geq 1$ [see Eq. (18)] puts a stronger limit on $R$ and so the bound is always obeyed. For instance, for $-1<$ $a \leq 0$ and $0<\eta \leq 1$, the bound is irrelevant. We have found that solutions where $-1<a \leq 0$ are thermodynamically stable solutions for all dimensions $d$. It is notable that stable solutions for all $d$ obey automatically the holographic entropy bound. If $\eta^{\frac{1}{d-2}} r_{+}^{a}>1$, then only those configurations of which $R$ obeys Eq. (62) are the ones that satisfy the bound.

\section{Entropy of the shell for large d}

When generalizing a physical system to higher dimensions, one should understand how the physical quantities, in particular the entropy, change with the dimension. In particular, for the entropy, this might have some implications on whether or not the system stays within one or both entropic bounds. In this connection, the $d \rightarrow \infty$ limit is useful and interesting. We will take the limit $d \rightarrow \infty$ and see how the entropy of a self-gravitating thin shell acts in response. To do so, we write the solid angle given in Eq. (8) in the following way, using the Stirling approximation: $\Omega_{d-2}=\sqrt{\frac{2}{e}}\left(\frac{2 \pi e}{d-3}\right)^{\frac{d-2}{2}}\left(1+O\left(\frac{1}{d}\right)\right)$. Although the approximation works better as $d \rightarrow \infty$, it is also a good fit for any $d \geq 4$. For the shell's entropy given in Eq. (32), we find

$$
S=\left(\frac{2 \pi e}{d}\right)^{d / 2} \eta r_{+}^{d(a+1)}
$$

Clearly, we have that $S \rightarrow 0$ as $d$ grows, and this is because the solid angle converges very quickly to zero, with $1 / d^{d / 2}$. Instead of setting $\eta$ as a constant, we could include the
$1 / d^{d / 2}$ factor into $\eta_{d} \equiv \eta / d^{d / 2}$ and set it as our problem's constant. But we will not do this here. One can also see how the large $d$ limit affects the distance to the holographic bound. Computing the ratio between the two, the solid angle terms cancel out, and we find $\frac{S}{A / 4}=\eta\left(\frac{r_{+}^{a+1}}{R}\right)^{d}$. Now, as mentioned previously, if $a \leq 0$ and $\eta \leq 1$, the bound is always satisfied, and we see that, as $d$ increases, the shell's entropy will distance itself farther from the bound; i.e., in this case, it holds that $\frac{S}{A / 4} \leq 1$.

We can additionally study the behavior of the other physical quantities of the shell. Since $(1-k)$ goes to zero with $\left(\frac{r_{+}}{R}\right)^{d}$ [see Eq. (19)], both the shell's rest mass $M$ and pressure $p$ go to zero, as one can check in Eqs. (21) and (22). Because $k \rightarrow 1$, the temperature is $T=1 / b$, and its behavior at the large $d$ limit depends on the sign of the equation of state exponent $a$, as can be seen in Eq. (31). For $a \leq 0$, the temperature $T$ diverges with $d r_{+}^{-a d}$, whereas for $a>0, T$ goes to zero in the $d \rightarrow \infty$ limit.

\section{BLACK HOLES IN $\boldsymbol{d}$ DIMENSIONS: ENTROPY, LOCAL THERMODYNAMIC STABILITY, SMARR FORMULA, ENTROPY BOUNDS, AND LARGE $d$}

\section{A. Black hole equation of state and entropy}

We are now interested in studying black hole properties in $d$ dimensions using the results from thin shells. For that, we take the $d$-dimensional shell to its gravitational radius $R \rightarrow r_{+}$, i.e., we take the quasiblack hole limit $[17,18]$. At this quasiblack hole stage the exterior spacetime to the shell is that of a $d$-dimensional Schwarzschild black hole, i.e., a Tangherlini black hole.

To do this, note that one possible equation of state for the temperature of the shell is the Hawking temperature $T_{+}$ given by $T_{+}=\frac{d-3}{4 \pi} \frac{1}{r_{+}}$[27]; i.e., the inverse temperature is $b_{+}=\frac{4 \pi}{d-3} r_{+}$. From Eq. (31) for the inverse temperature $b\left(r_{+}\right)$of the shell at infinity, one sees that putting $a=0$ and $\eta=1$, one recovers precisely $b_{+}$. In this case, from Eq. (32), the entropy $S$ of the shell with radius $R$ is $S=\frac{1}{4} A_{+}$. We now can take the limit and send the radius $R$ of the shell to its own gravitational radius $r_{+}, R \rightarrow r_{+}$.

Before we do that, we note that when performing the quasistatic collapse of the shell into $r_{+}$, the only reasonable equation of state for the inverse temperature is indeed $b_{+}$. The analysis we have been doing demands thermal equilibrium so that we can safely use the first law of thermodynamics (23). If then we take into account that quantum fields are present just outside the shell at its own gravitational radius $R=r_{+}$, the shell's inverse temperature must be the black hole inverse temperature, so $b\left(r_{+}\right)$in Eq. (31) must have the expression $b\left(r_{+}\right)=b_{+}=\frac{4 \pi}{d-3} r_{+}$in order to have equilibrium. Moreover, it has been shown in some particular instance [34] that the thermal energy-momentum tensor $T_{b}^{a}$ for a field at temperature $T_{\text {field }}$ is of the form [34] 
$T_{b}^{a}=\frac{T_{\text {field }}^{4}-T_{+}^{4}}{g_{00}^{2}} f_{b}^{a}$, for some tensor $f_{b}^{a}$ finite at the horizon, with $g_{00}$ being the time-time metric component of the static spacetime. Assuming this is also valid in $d$ dimensions, we see that, since $g_{00}$ is zero at $R=r_{+}, T_{b}^{a}$ diverges unless the temperature of the field $T_{\text {field }}$ is the Hawking temperature $T_{+}, T_{\text {field }}=T_{+}$, and so $b_{\text {field }}=b_{+}$.

So, when one collapses the shell quasistatically into a black hole, i.e., $R=r_{+}$, Eq. (31) must take the form

$$
b_{+}\left(r_{+}\right)=\frac{4 \pi}{d-3} r_{+} .
$$

Then, the entropy from Eq. (32) is

$$
S_{+}=\frac{1}{4} A_{+} .
$$

This is the Bekestein-Hawking entropy in $d$ dimensions, obtained here from the self-gravitating shell formalism with the input of the Hawking temperature.

\section{B. Black hole intrinsic thermodynamic stability}

For a black hole, $a=0$ and $\eta=1$. In the black hole limit, we take in addition $R \rightarrow r_{+}$implying $k \rightarrow 0$. The stability equations we are interested in are given in Eqs. (40), (45), and (51). We first take $a=0$ in the stability conditions and see the properties for the shell with this $a$. Then, we take the black hole limit $R \rightarrow r_{+}$and discuss the features in this case.

For a fixed shell's area $A, a=0$, and $R \rightarrow r_{+}$, thermodynamic stability is taken from Eq. (40) or Eq. (43). Clearly, one finds that the shell is thermodynamically stable at the gravitational radius for $d \geq 4$. Indeed, when the shell with $a=0$ is at its gravitational or horizon radius, i.e., $k=0$, it satisfies marginally the intrinsic thermodynamic stability criterion (40). This is because the heat capacity $C_{A}=T^{-2}\left(\frac{\partial^{2} S}{\partial M^{2}}\right)_{A}^{-1}$ goes to zero with $T^{-2}$ in this limit. Since $C_{A}$ is also defined as $C_{A}=\left(\frac{\partial M}{\partial T}\right)_{A}, C_{A}=0$ means that the mass of the shell cannot be altered by any change on the infinitely high temperature. In this limit, we cannot increase the mass $M$ of the shell with $A=A_{+}$, i.e., $R=r_{+}$, constant, since from Eq. (21) in this limit one has $\frac{\gamma}{2} M=r_{+}^{d-3}$. So, to change $M$, one has to change the radius $R=r_{+}$. Moreover, York's results for black holes in a canonical ensemble [6] imply that when the heat reservoir is placed at $R=r_{+}$, the black hole is thermodynamically marginally stable. The two results are indeed the same as the two situations deal with the same black hole, namely, a black hole in a heat reservoir at its horizon at temperature $T$. So, York's heat reservoir at the black hole horizon and the massive shell at the gravitational radius are the same thing, and York's criterion for thermodynamic stability is precisely reproduced.

For fixed proper mass $M, a=0$, and $R \rightarrow r_{+}$, thermodynamic stability comes from Eq. (45), and we have seen that the $a=0$ shell is stable under this condition for all radii, in particular for $R=r_{+}$.

For free $A$ and $M, a=0$, and $R \rightarrow r_{+}$, thermodynamic stability comes from Eq. (51). Since in this case $k_{3}=0$ as we have seen, the only stable case is precisely $R=r_{+}$; i.e., the black hole is stable.

\section{Smarr formula}

For a black hole, $a=0$, and the black hole Euler relation has to be taken from Eq. (56) by putting $a=0$. In the black hole limit, we take in addition $R \rightarrow r_{+}$, implying $k \rightarrow 0$. Since both the temperature $T$ and pressure $p$ go with $k^{-1}$, one has $k M=\frac{d-2}{d-3} T_{+} S_{+}-\frac{d-2}{d-3} p_{+} A_{+}$, where $T_{+}=1 / b_{+}$ is the Hawking temperature with $b_{+}$given in Eq. (64), $S_{+}$is the Bekenstein-Hawking entropy given in Eq. (65), $p_{+}$is the redshifted pressure $p_{+}=p k$ with $p$ given in Eq. (22), and $A_{+}$is the horizon area given in Eq. (10). So, since $k \rightarrow 0$ in this limit, this translates into $0=\frac{d-2}{d-3} T_{+} S_{+}-$ $\frac{(d-2) \Omega_{d-2}}{16 \pi} r_{+}^{d-3}$, which upon using Eqs. (7) and (9) yields $0=\frac{d-2}{d-3} T_{+} S_{+}-m$, i.e.,

$$
m=\frac{d-2}{d-3} T_{+} S_{+},
$$

the Smarr formula for a black hole in $d$ dimensions; see also Ref. [28]. In four dimensions, this is $m=2 T_{+} S_{+}$, the original Smarr formula [1].

Smarr formula in $d$ dimensions has been provided before, but it is remarkable that one can derive it from the shell mechanics and thermodynamics in a nontrivial way. The rest mass term $M$ that surely appears in the Euler relation for the shell gives no contribution, and it is the term $p A$ that contains the spacetime energy and thus yields the mass $m$ term. This is in line with the black hole mass formula derived in Ref. [21] for $d=4$ using the membrane paradigm approach, where the term $2 p_{+}$is indeed the usual black hole surface gravity $\kappa$ divided by $4 \pi$ and was shown to be also the horizon surface energy density $\sigma$ measured at infinity [21]. When $\sigma$ is multiplied by $A_{+}$one obtains the total energy $m$.

\section{Other topics on black hole entropy}

\section{Bekenstein entropy bound for the d-dimensional black hole}

Let us now take the $d$-dimensional Bekenstein bound, Eq. (58), in the black hole limit $R \rightarrow r_{+}$. For the shell, this bound is provided in Eq. (59) if we choose $E=M$ and in Eq. (60) if we choose $E=m$.

For $E=M$, the bound Eq. (59) is then

$$
S_{E}<2 S_{+},
$$


where $S_{+}=\frac{1}{4} A_{+}$is the black hole entropy given in Eq. (65).

For $E=m$, the bound Eq. (60) is

$$
S_{E}<S_{+} \text {. }
$$

Which case shall we choose, $E=M$ or $E=m$ ? If we stick to $S_{E}<S_{+}$, i.e., to the statement that the maximum entropy for an area $A$ is when there is a black hole in this area, then we should have Eq. (68), and so we should choose $E=m$; see also Ref. [9]. But all this relies on our previous choice of $\alpha$, and so the argument is only of heuristic value.

\section{Holographic entropy bound for the d-dimensional black hole}

When the holographic entropy bound of Eq. (61) is applied to the shell, we get Eq. (62). In the particular case in which the shell is a black hole, then $a=0$ and $\eta=1$, and we get that the bound is satisfied for any $R \geq r_{+}$: This means that in this case all the shells, including the black hole limit, satisfy the bound. That the black hole satisfies the bound is expected, since black holes pose the highest entropy outcome from gravitational collapse.

\section{Entropy of the black hole for large d}

The entropy of a self-gravitating shell for large $d$ is given in Eq. (63). For the black hole case, one puts $a=0$ and $\eta=1$ to obtain

$$
S_{+}=\frac{1}{d^{d / 2}} r_{+}^{d} .
$$

Note that $S_{+}$is still $S_{+}=\frac{1}{4} A_{+}$but in the large $d$ limit, one has $r_{+} \rightarrow 0, A_{+} \rightarrow 0$, so the entropy of the black holes vanishes in the large $d$ limit.

\section{CONCLUSIONS}

The first law of thermodynamics on a $d$-dimensional self-gravitating spherical thin shell is used in its entropy representation, where the entropy is a function of the shell's rest mass and the shell's area $A$ or, since $A=4 \pi R^{2}$, the shell's radius, $S=S(M, R)$. The pressure equation of state $p=p(M, R)$ is fixed by the spacetime junction conditions, and the inverse temperature equation of state $\beta=\beta(M, R)$ must have the form $\beta=k\left(r_{+}, R\right) b\left(r_{+}\right)$, with $b\left(r_{+}\right)$arbitrary, in order to satisfy the integrability condition for the entropy, where $r_{+}=r_{+}(M, R)$. Integrating the first law, we find that the shell's entropy is given as a function of the gravitational radius $r_{+}(M, R)$ alone.

With the inverse temperature equation of state now controlled completely by $b\left(r_{+}\right)$, we specify a power-law equation for $b\left(r_{+}\right)$with its exponent governed by a parameter $a$ and such that when $a=0$ the inverse temperature $b\left(r_{+}\right)$has the Hawking form. The thermodynamic stability conditions can be worked out generically, and, in particular, for $a=0$, it is found that the shell is stable when its radius is in between its own gravitational radius and the photonic radius, i.e., the radius of circular photon orbits, reproducing unexpectedly York's thermodynamic stability criterion for a $d=4$ black hole in a heat reservoir canonical ensemble. Since the two systems are different, this is an unexpected result and hints that what is important for thermodynamic stability is the place of the shell alone, whether it is a heat reservoir massless shell or a massive shell. An Euler formula for the matter is derived.

When put at its own gravitational radius, the shell spacetime turns into a black hole spacetime. In this limit, it is mandatory that the self-gravitating shell is at the Hawking temperature, which in turn renders through the formalism developed here, the Bekenstein-Hawking entropy in $d$ dimensions. The black hole is marginally stable as the heat capacity is zero. In this case, the physical situation is the same as in the $d=4$ York case; York's heat reservoir shell and the massive shell at the gravitational radius are the same thing, and so York's criterion for marginal stability is precisely reproduced. The Smarr formula for black holes pops out naturally and surprisingly.

\section{ACKNOWLEDGMENTS}

R. A. acknowledges support from the Doctoral Programme in the Physics and Mathematics of Information (DP-PMI) and the Fundação para a Ciência e Tecnologia (FCT Portugal) through Grant No. PD/BD/ 135011/2017. J.P. S. L. acknowledges FCT for financial support through Project No. UID/FIS/00099/2013 and Grant No. SFRH/BSAB/128455/2017 and Coordenação de Aperfeiçoamento do Pessoal de Nível Superior (CAPES), Brazil, for support within the Programa CSFPVE, Grant No. 88887.068694/2014-00. G. Q. acknowledges the support of the Fundação para a Ciência e Tecnologia (FCT Portugal) through Grant No. SFRH/ BD/92583/2013. 
[1] L. Smarr, Mass Formula for Kerr Black Holes, Phys. Rev. Lett. 30, 71 (1973).

[2] J. M. Bardeen, B. Carter, and S. W. Hawking, The four laws of black hole mechanics, Commun. Math. Phys. 31, 161 (1973).

[3] J. D. Bekenstein, Black holes and entropy, Phys. Rev. D 7, 2333 (1973).

[4] S. W. Hawking, Particle creation by black holes, Commun. Math. Phys. 43, 199 (1975).

[5] J. B. Hartle and S. W. Hawking, Path-integral derivation of black-hole radiance, Phys. Rev. D 13, 2188 (1976).

[6] J. W. York, Black hole thermodynamics and the Euclidean Einstein action, Phys. Rev. D 33, 2092 (1986).

[7] H. W. Braden, J. D. Brown, B. F. Whiting, and J. W. York, Charged black hole in a grand canonical ensemble, Phys. Rev. D 42, 3376 (1990).

[8] C. S. Peça and J. P. S. Lemos, Thermodynamics of ReissnerNordström-anti-de Sitter black holes in the grand canonical ensemble, Phys. Rev. D 59, 124007 (1999).

[9] E. A. Martinez, Fundamental thermodynamical equation of a self-gravitating system, Phys. Rev. D 53, 7062 (1996).

[10] J. P. S. Lemos, G. M. Quinta, and O. B. Zaslavskii, Entropy of a self-gravitating electrically charged thin shell and the black hole limit, Phys. Rev. D 91, 104027 (2015).

[11] J. P. S. Lemos, G. M. Quinta, and O. B. Zaslavskii, Entropy of an extremal electrically charged thin shell and the extremal black hole, Phys. Lett. B 750, 306 (2015).

[12] J. P. S. Lemos, G. M. Quinta, and O. B. Zaslavskii, Entropy of extremal black holes: Horizon limits through charged thin shells in a unified approach, Phys. Rev. D 93, 084008 (2016).

[13] E. A. Martinez and J. W. York, Additivity of the entropies of black holes and matter in equilibrium, Phys. Rev. D 40, 2124 (1989).

[14] P. C. W. Davies, L. H. Ford, and D. N. Page, Gravitational entropy: Beyond the black hole, Phys. Rev. D 34, 1700 (1986).

[15] W. A. Hiscock, Gravitational entropy of nonstationary black holes and spherical shells, Phys. Rev. D 40, 1336 (1989).

[16] F. Pretorius, D. Vollick, and W. Israel, An operational approach to black hole entropy, Phys. Rev. D 57, 6311 (1998).

[17] J. P. S. Lemos and O. B. Zaslavskii, Entropy of quasiblack holes, Phys. Rev. D 81, 064012 (2010).

[18] J. P. S. Lemos and O. B. Zaslavskii, Entropy of extremal black holes from entropy of quasiblack holes, Phys. Lett. B 695, 37 (2011).
[19] R. H. Price and K. S. Thorne, Membrane viewpoint on black holes: Properties and evolution of the stretched horizon, Phys. Rev. D 33, 915 (1986).

[20] J. P. S. Lemos and O. B. Zaslavskii, Membrane paradigm and entropy of black holes in the Euclidean action approach, Phys. Rev. D 84, 064017 (2011).

[21] J. P.S. Lemos and O.B. Zaslavskii, Black hole mass formula in the membrane paradigm, Phys. Rev. D 97, 064008 (2018).

[22] J. P. S. Lemos and G. M. Quinta, Entropy of thin shells in a $(2+1)$-dimensional asymptotically AdS spacetime and the BTZ black hole limit, Phys. Rev. D 89, 084051 (2014).

[23] J. P. S. Lemos, F. J. Lopes, M. Minamitsuji, and J. V. Rocha, Thermodynamics of rotating thin shells in the BTZ spacetime, Phys. Rev. D 92, 064012 (2015).

[24] J. P. S. Lemos, M. Minamitsuji, and O. B. Zaslavskii, Thermodynamics of extremal rotating thin shells in an extremal BTZ spacetime and the extremal black hole entropy, Phys. Rev. D 95, 044003 (2017).

[25] J. P. S. Lemos, M. Minamitsuji, and O. B. Zaslavskii, Unified approach to the entropy of an extremal rotating BTZ black hole: Thin shells and horizon limits, Phys. Rev. D 96, 084068 (2017).

[26] F. C. P. Monteiro, General and Specific Results for d-Dimensional Orbits in General Relativity: From a FourDimensional Spacetime to the Large d Limit, M.Sc. thesis, Instituto Superior Técnico, Lisbon, 2015.

[27] C. M. Harris and P. Kanti, Hawking radiation from a $(4+n)$-dimensional black hole: Exact results for the Schwarzschild phase, J. High Energy Phys. 10 (2003) 014.

[28] R. Banerjee, B. R. Majhi, S. K. Modak, and S. Samanta, Killing symmetries and Smarr formula for black holes in arbitrary dimensions, Phys. Rev. D 82, 124002 (2010).

[29] H. B. Callen, Thermodynamics and an Introduction to Thermostatistics (Wiley, New York, 1985).

[30] J. D. Bekenstein, Entropy bounds and the second law for black holes, Phys. Rev. D 27, 2262 (1983).

[31] G. 't Hooft, Dimensional reduction in quantum gravity, in Salamfestschrift, A Collection of Talks from the Conference on Highlights of Particle and Condensed Matter Physics, edited by A. Ali et al. (World Scientific, Singapore, 1994).

[32] L. Susskind, The world as a hologram, J. Math. Phys. (N.Y.) 36, 6377 (1995).

[33] S. Hod, Holographic entropy bound in higher-dimensional spacetimes, Phys. Rev. D 97, 126012 (2018).

[34] D. J. Loranz, W. A. Hiscock, and P. R. Anderson, Thermal divergences on the event horizons of two-dimensional black holes, Phys. Rev. D 52, 4554 (1995). 